International Journal of Structural Stability and Dynamics Vol. 18, No. 8 (2018) 1840007 (24 pages)

\title{
Nonlinear Characterization of the MRE Isolator using \\ Binary-Coded Discrete CSO and ELM
}

\author{
(C) World Scientific Publishing Company
}

DOI: $10.1142 /$ S0219455418400072

\author{
Yang $\mathrm{Yu}^{*}$, Yancheng $\mathrm{Li}^{*}$,
} Sayed Royel ${ }^{\dagger}$

${ }^{*}$ School of Civil and Environmental Engineering University of Technology Sydney NSW 2007, Australia

${ }^{\dagger}$ School of Electrical, Mechanical and Mechatronic Systems University of Technology Sydney, NSW 2007, Australia

†Yancheng.li@uts.edu.au

\$Jianchun.li@uts.edu.au.

Received 27 March 2017

Accepted 2 July 2017 Published

\begin{abstract}
Magnetorheological elastomer (MRE) isolator has been proved as a promising semi-active control device for structural vibration control. For its engineering application, developing an accurate and robust model is definitely necessary and also a challenging task. Most of the present models, belonging to parametric models, need to identify various model parameters and sometimes are not capable of perfectly capturing the unique characteristics of the device. In this work, a novel nonparametric model is proposed to characterize the inherent dynamics of the MRE isolator with the features of hysteresis and nonlinearity. Initially, dynamic tests are conducted to evahuate the performance of the isolator under various loading conditions, including harmomic, random, and seismic excitations. Then, on the basis of the captured experimental results, a hybrid learning method is designed to forecast the nonlinear responses of the device with known external inputs. In this method, a type of single hidden layer feed-forward network, called extreme learning machine (ELM), is developed to forecast the nonlinear responses (shear force) of the device with captured velocity, displacement, and current level. To obtain optimal performance of the developed model, an improved binary-coded discrete cat swarm optimization (BCDCSO) method is adopted to select optimal inputs and neuron number in the hidden layer for the network development. The performance of the proposed method is verified through the comparison between experimental results and model predictions. Due to the noise influence in the practical condition, the robustness of the proposed method is also validated via adding noise disturbance into the supplying currents. The results show that the proposed method outperforms the standard ELM in terms of characterization of the MRE isolator, even though the captured responses are polluted with external measurement noises.
\end{abstract}

Keywords: MRE isolator; nonlinear dynamics; extreme learning machine; cat swarm optimization.

I Corresponding authors. 


\section{Introduction}

Magnetorheological elastomer (MRE) is a type of intelligent material, the mechanical properties of which are continuously, quickly, and reversibly controlled via external applied magnetic fields. ${ }^{1-3}$ Based on this merit, MRE has been widely applied to design semi-active devices to realize stiffness and damping variability for vibration control of engineering structures against hazard external vibrations. ${ }^{4,5}$ Hoang et al. developed a tuned vibration absorber (TVA) based on MRE for powertrain vibration suppression. ${ }^{6,7}$ Ginder et al. utilized MREs to develop an adjustable automotive bushing for vibration reduction on the rotational and translational movements of vehicle wheels. ${ }^{8}$ Du et al. proposed a novel isolator based on MRE, which is employed for seat vibration control in vehicles, and the developed MRE isolator is able to work in both compressive and shear modes. ${ }^{9}$ Sun et al. also developed the multi-layer MRE isolators, with the feature of negative changing stiffness, used for the horizontal vibration reduction of the vehicle seats. ${ }^{10}$ In the application field of civil engineering, $\mathrm{Li}$ et al. prototyped the first adaptive seismic isolator for earthquake mitigation of building structures using MREs. ${ }^{11}$ In this design, a laminated structure with 47 layers of MRE sheets and 46 layers of steel sheet was adopted. The experimental results indicated that under the applied magnetic fields from 0 to $0.3 \mathrm{~T}$, the increases of the shear force, lateral stiffness, and damping ratio of the device can be up to $45 \%$, $38 \%$, and $11.2 \%$, respectively. Then, to achieve higher MR effect on MRE-based isolator, Li et al. developed another adaptive base isolator using soft MREs, which is able to contribute to $1630 \%$ stiffness increase and $1480 \%$ force increase when the device is supplied with the electricity currents from 0 to $3 \mathrm{~A} \cdot{ }^{12}$ This amazing result validated the potential and feasibility of development of intelligent base isolation system using MRE isolators.

Although MRE isolators are significantly promising in the field of structural vibration control, the major aporia that affects their practical implementations is the modeling of their unique dynamics with high nonlinearity and inherent hysteresis. To date, several MRE isolator models have been reported in the literatures based on various methods. The models gained via the conclusive method belongs to the parametric models, including Kelvin-Voigt model, Bouc-Wen hysteresis-based models, strain stiffening model, simplified hysteresis model, etc. Yang et al. adopted the Kelvin-Voigt model to portray the nonlinear responses of a MRE isolator in the mixed mode of shear and compression. ${ }^{13}$ To improve the Kelvin-Voigt model, Li et al. developed a strain stiffening model for characterizing MRE isolator, consisting of a standard three-parameter solid model and a modified Maxwell model connected in parallel. ${ }^{12}$ Based on the same device, Yang et al. utilized the classical Bouc-Wen hysteresis-based model to illustrate the shear force response of the device, in which a highly nonlinear differential equation is employed to express the hysteresis with extravagant complexity. ${ }^{14}$ They also analyzed the effects of model parameters on the shape of the hysteretic force-displacement loops. Behrooz et al. developed a modified Bouc-Wen hysteresis model for MRE-based variable stiffness and damping isolator, 
in which a current dependent three-parameter solid model is combined with the standard Bouc-Wen model. ${ }^{15}$ However, this improved model has a total of 14 parameters to be identified, which is hard to implement in practice. To avoid a large number of parameters and differential equations in existing models, Yu et al. utilized the hyperbolic sine function (HSF) to substitute for differential equations to depict the hysteresis, making the model easy to be identified. However, the HSF is not able to accurately describe the phenomenon of stiffness hardening when the device is supplied with higher currents. In short, because of large number of parameters and highly nonlinear differential equations, the correspondent models for demonstrating inverse dynamics of MRE isolators are challenging to obtain. As a result, existing parametric models of MRE isolators are difficult to emerge into present control configurations.

On the other hand, the soft computing techniques contribute to the development of nonparametric models for MRE isolators, in which the dynamical behavior of the device cannot be described by the specific mathematical expressions. So far, the nonparametric modeling on MRE isolators is rarely investigated. The only model that has been reported is based on artificial neural networks (ANN), which are used to implicitly forecast the complicated nonlinear relationship between dependent and independent variables. In Ref. 16, Fu et al. proposed a nonlinear auto-regressive exogenous (NARX)-based recurrent neural networks to predict the dynamical features of a MRE isolator in shear-compression mode. The proposed network has a three-layer configuration, in which the displacement, velocity, command current and their historical values, and the historical force are used as inputs and the present force is the network output. Then, the trial-and-error method is adopted to choose detailed inputs and number of hidden neuron. Although the trained model provides a satisfactory performance on tracking the nonlinearity of force-velocity response, two time delays in the input neurons lead to a complicated network structure, which will increase the calculation time. Besides, the data used for model training and validation in Ref. 16 is based on the fixed excitation frequency. However, in practice, the loading frequency is always changeable. Consequently, the performance of the proposed NARX network to model the MRE isolator with variable excitation frequencies should be further investigated. Furthermore, when the MRE isolators are used for the protection of the civil structures, the measured information is unavoidably polluted with environmental and measurement noises. If the captured noisy data is directly used for the model training, the generalization ability of the obtained network will be greatly affected. Although noise filters are effective tools to eliminate the influences caused by noises, they are out of operation when the noises have the same frequency range as the measurement signals. Accordingly, it is also significant to investigate the influence of noises on the prediction capacity of the trained model.

In this paper, a hybrid modeling method is proposed to characterise the nonlinear dynamics of MRE isolators. First of all, the dynamical performance of a prototype of MRE isolator is evaluated under various loading conditions, including sinusoidal, 
random and seismic excitations, and different electricity current inputs. Then, based on measured responses of the device and current levels applied to the device, an ELM-based model is built to portray the complex input-output relationship of the network, in which the network inputs are displacement, velocity and electricity current at current and previous time instants, and previous force instants and the network output is the current shear force. Since the over-fitting problem may occur due to a large number of network inputs, it is preferable to logically select the network inputs prior to the model training. In this work, a novel binary-coded cat swarm optimization algorithm (BCDCSO) algorithm is proposed to optimize the input subsets of the ELM. Then, the performance of this optimal ELM is evaluated via the comparison between experimental data and network predictions. Moreover, the noise disturbances are added to the applied current, which is used to assess the robustness of the proposed model. The results show that the ELM with BCDCSOoptimized inputs and hidden neurons outperforms the analogous learning method (ELM) in terms of model accuracy.

\section{Preliminaries}

\subsection{MRE isolator}

As a novel smart material, MRE has the benefits of both MR material and elastomer, i.e. quick response, controllable and reversible mechanical property. Generally, the MRE is made of three principle ingredients: elastomer matrix, polarized magnetic particles, and silicon oils. When the MRE is supplied with external magnetic field, its mechanical properties, such as elasticity modulus and damping, will be greatly improved. It is able to avoid the problems of poor stability, sedimentation, and particle wearing existing in MR fluid. Because of this inherent feature, the MRE exhibits the huge potentiality in the field of vibration and shock mitigation of mechanical or civil infrastructure.

Based on the advantages of the MRE material, a new adaptive isolator was developed by $\mathrm{Li}$ et al. using a traditional laminated configuration of rubber bearing. ${ }^{11,12}$ To realize a high performance of MR effect, the soft MRE material was employed in the design of the device. Fig. 1(a) shows the design schematic of the MRE isolator while Fig. 1(b) is the corresponding photo of real-product. It is seen from the figure that the device design is achieved via the replacement of conventional rubbers with MREs. The core part of the device is the formation of the laminated configuration via alternative vulcanization of MRE sheets and steel plates. There are a total of 25 layers of MRE sheet and 26 layers of steel plate in the design with the uniform $1 \mathrm{~mm}$ thickness. In the core, the steel plate provides the loading capacity to the isolator in the vertical direction while the MRE sheet provides high horizontal flexibility to the device. Besides, an electromagnetic coil is installed surrounding the core with certain gap (allowing lateral deformation) to produce uniform magnetic flux running through the MRE sheet. As a consequence, the stiffness property of the 


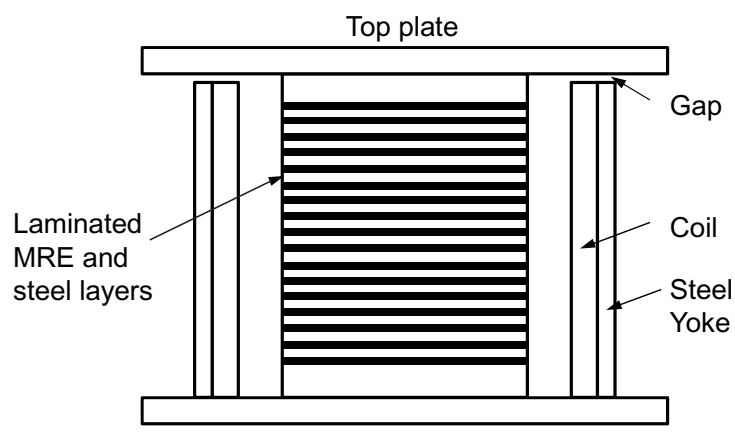

(a)

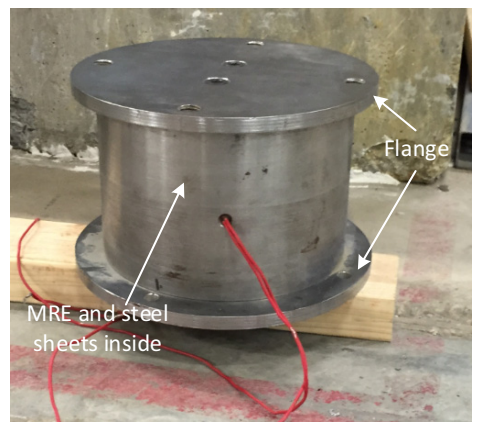

(b)

Fig. 1. Structure of the MRE isolator (a) cross-sectional view and (b) real-object photo.

device can be properly adjusted via changing the applied current of solenoid. The gap between the coil and core offers the device with the maximal shear deformation of $15 \mathrm{~mm}$. The core and coil are incorporated with the steel wall, which is the outer layer of the device. The core is fixed to both top and bottom plates of the device while the coil and steel yoke is fixed to the bottom plate. The minimal axial loading capacity of the device is $50 \mathrm{~kg}$ under maximal shear deformation without the applied current. ${ }^{12}$ With increase of the applied current, the axial load carrying capacity will be increased simultaneously. When the shear deformation is less than $15 \mathrm{~mm}$, the axial load carrying capacity of the device will also be improved significantly. Because it is the function of shear modulus of the material and the thickness of the cross-sectional thickness, the axial loading capacity can be designed based on the practical application requirement.

\subsection{Extreme learning machine}

ELM is a kind of single-hidden layer feed-forward network (SLFN). Compared with traditional learning theories, ELM adopts the stochastic strategy to assign the input weights and thresholds of the network and the regularization calculation to obtain the network output weights. ${ }^{17,18}$ The network in such a model is still capable of approximating any continuous system and simultaneously avoiding the adjustment of all the network parameters. Accordingly, ELM has faster training speed, fewer training error, and stronger generalization capacity than conventional neural network. The basic principle of ELM can be summarized as follows.

Suppose that the SLFN has $l$ input neurons, $m$ hidden neurons, and a linear output neuron, shown in Fig. 2. The following equation can be used to depict the relationship between network inputs and output:

$$
F_{m}(x)=\sum_{k=1}^{m} w_{k} L\left(\alpha_{k}, \beta_{k}, x\right)
$$


Y. Yu et al.

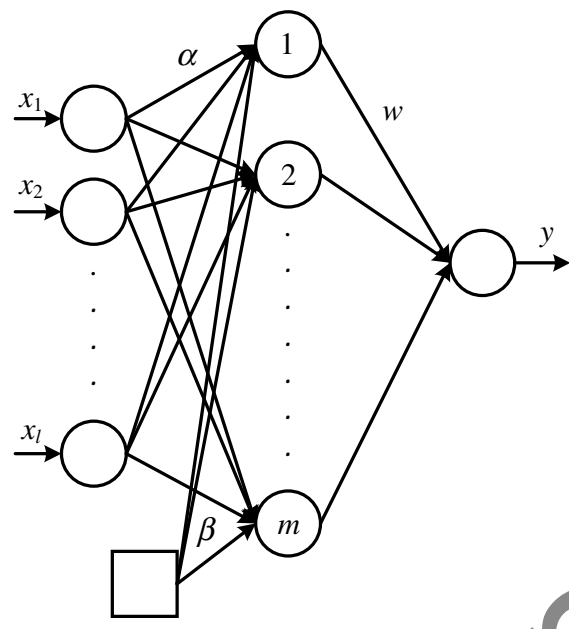

Fig. 2. Configuration of ELM with $l$ inputs, $m$ hidden nodes and a output.

where $\alpha_{k}$ and $\beta_{k}$ denote the input weights and thresholds of the $k$ th neuron in the hidden layer, respectively; $w_{k}$ denotes the connection weight between $k$ th neuron in the hidden layer and output neuron; $L\left(\alpha_{k}, \beta_{k}, x\right)$ represents the $k$ th output in the hidden layer using the stochastic parameter values $\left(\alpha_{k}, \beta_{k}\right)$.

In ELM, suppose that there is a group of training samples $\left(x_{i}, y_{i}\right)(i=$ $1,2, \ldots, M, M$ is the total number of training samples) and the output model of ELM can be represented using the following equation:

$$
F_{m}\left(x_{i}\right)=\sum_{k=1}^{m} w_{k} L\left(\alpha_{k}, \beta_{k}, x_{i}\right), \quad i=1,2, \ldots, M .
$$

During the model training, parameters $w, \alpha$, and $\beta$ should satisfy the relationship as follows:

$$
\sum_{k=1}^{m} w_{k} L\left(\alpha_{k}, \beta_{k}, x_{i}\right)=y_{i}
$$

Equation (3) could be represented using the form of matrix:

$$
G w=Y,
$$

where $G$ denotes the network output matrix in the hidden layer and

$$
G=\left[\begin{array}{ccc}
L\left(\alpha_{1}, \beta_{1}, x_{1}\right) & \cdots & L\left(\alpha_{m}, \beta_{m}, x_{1}\right) \\
L\left(\alpha_{1}, \beta_{1}, x_{2}\right) & \cdots & L\left(\alpha_{m}, \beta_{m}, x_{2}\right) \\
\vdots & \vdots & \vdots \\
L\left(\alpha_{1}, \beta_{1}, x_{M}\right) & \cdots & L\left(\alpha_{m}, \beta_{m}, x_{M}\right)
\end{array}\right],
$$

$w=\left[w_{1}, w_{2}, \ldots, w_{m}\right]^{T}, Y$ denotes the model targets and $Y=\left[y_{1}, y_{2}, \ldots, y_{M}\right]^{T}$. 
To calculate the unknown output vector $w$, an error function Err of ELM is first defined as follows:

$$
\operatorname{Err}(w)=\sum_{i=1}^{M}\left|Y_{i}-y_{i}\right|^{2} .
$$

The training objective of ELM is to search for the optimal vector $w$ to guarantee the minimum value of error between network outputs and real values of training samples, and the corresponding optimization problem is provided as:

$$
\min \operatorname{Err}(w)=\min _{w}\|Y-\hat{Y}\|_{2}=\min _{w}\|G w-\hat{Y}\|_{2},
$$

where $Y$ and $\hat{Y}$ denote the network outputs and practical measured values, respectively. In accordance with Ref. 17, when the activation function in the hidden layer is infinitely differential, the input weights $\alpha_{k}$ and the thresholds $\beta_{k}$ is capable of being randomly assigned. Hence, it is unnecessary to adjust their values and the output matrix $G$ is also constant during the network training. The training procedure of ELM can be regarded as calculating least square solution $\hat{w}$ of linear system $G w=Y$ :

$$
\|G \hat{w}-Y\|_{2}=\min _{w}\|G w-Y\|_{2}
$$

If the neuron number in the hidden layer is equal to that of the training samples, the network will approximate to the training samples without any error for arbitrary $\alpha_{k}$ and $\beta_{k}$. If the neuron number in the hidden layer is smaller than that of training samples, the training error will approximate to an arbitrarily small positive constant $\varepsilon$. In this case, the matrix $G$ is not a square matrix and the linear system cannot be satisfied with suitable $w, \alpha$, and $\beta$. Accordingly, the smallest norm least square solution $\tilde{w}$ is utilised to substitute for $w$ in the linear system:

$$
\tilde{w}=G^{*} Y
$$

where $G^{*}$ is the Moore Penrose pseudo-inverse of $G$.

\subsection{Cat swarm optimization}

CSO is a newly developed swarm optimization algorithm that simulates the food hunting behavior of cats. Different from other homogeneous animals, the cat spends most of its time on resting and observing the target, which can be defined as searching behavior. ${ }^{19}$ After the cat detects the prey, it will rapidly move and chase it, which can be depicted as tracking behavior. In CSO, two modes of operations are mathematically demonstrated to deal with the complicated optimization problem.

\subsubsection{Searching mode}

In the searching mode, there are four elements that should be introduced first:

(1) Searching memory pool (SMP). SMP is defined as the size of searching memory of each cat, which indicates the location point searched by each cat. 
(2) Change rate of selected dimensions (CRD). CRD is defined as the change rate of selected area. In this mode, change range of each dimension is determined by CRD.

(3) Change number of dimensions (CND). CND is designed to indicate the number of dimensions that will be mutated in future, the value of which is randomly selected between 0 and maximum dimension.

(4) Self-location judgment (SLJ). SLJ is defined as the Boolean flag, which represents whether the location of the cat moving to is one of the previous locations or not.

The procedure of search mode can be summarized as the following five steps:

Step 1. Duplicate $n p(n p=S M P)$ copies of present location of each cat in SMP, i.e. $\mathrm{np}$ is the size of SMP. If the flag of SLJ is true, set $n p=\mathrm{SMP}-1$ and consider the current location of each cat as one of the candidates.

Step 2. For each copy in SMP, randomly add or subtract CRD\% of current values as updated values to replace the previous ones according to the CND.

Step 3. Calculate the fitness values of all the candidates.

Step 4. If all the fitness values of candidates are not completely same, evaluate the selection probability of each location point using the following equation:

$$
p_{i}=\frac{\left|\mathrm{Fit}_{i}-\mathrm{Fit}_{b}\right|}{\mathrm{Fit}_{\text {max }}-\mathrm{Fit}_{\text {min }}},
$$

where Fit $_{i}$ denotes the fitness value of $i$ th candidate and $0<i<$ np. $\mathrm{Fit}_{b}=\mathrm{Fit}_{\max }$ for the minimum optimization problem and $\mathrm{Fit}_{b}=\mathrm{Fit}_{\text {min }}$ for the maximum optimization problem. Or else, assign the selection probability of each candidate as 1 .

Step 5. Adopt the roulette wheel method to select one candidate and substitute the selection for the eurrent location.

\subsubsection{Tracking mode}

Tracking mode is developed for simulating the cat in tracking the target. In this mode, the movement of each cat is set according to the velocity of each dimension and the optimal location explored by the cat swarm. The procedure of tracking mode can be illustrated as follows:

Step 1. Update the velocity of each dimension using the following equation:

$$
v_{k, d}=v_{k, d}+r_{1} \cdot c_{1} \cdot\left(x_{\mathrm{opt}, d}-x_{k, d}\right), \quad d=1,2, \ldots, N_{d},
$$

where $x_{\mathrm{opt}, d}$ is the optimal location of the cat swarm; $x_{k, d}$ denotes the location of $k$ th cat in $d$ th dimension and $N_{d}$ is the total number of the dimensions; $c_{1}$ denote the constant; $r_{1}$ denotes a random number between 0 and 1 .

Step 2. Check whether the current velocity is out of range. If so, assign the velocity as the limitation value.

Step 3. Update the location of $i$ th cat in $d$ th dimension using the following equation:

$$
x_{k, d}=x_{k, d}+v_{k, d}
$$




\subsubsection{Procedure of $C S O$}

CSO combines the searching mode and tracking mode to solve the global optimization problem. First, a mixture probability (MP) is defined to determine the percentages of the cat swarm in both modes. Because the cats spend most of their time on observing (searching mode) rather than capturing the food (tracking mode), the MP is always set as a small value to satisfy the real situation. The detailed algorithm procedure is provided as follows (also shown in Fig. 3):

Step 1. Generate $N_{\text {pop }}$ cats in the swarm.

Step 2. $N_{\text {pop }}$ cats are randomly placed into $N_{d}$-dimensional solution space and are assigned with the random values in the velocity range. Then, according to

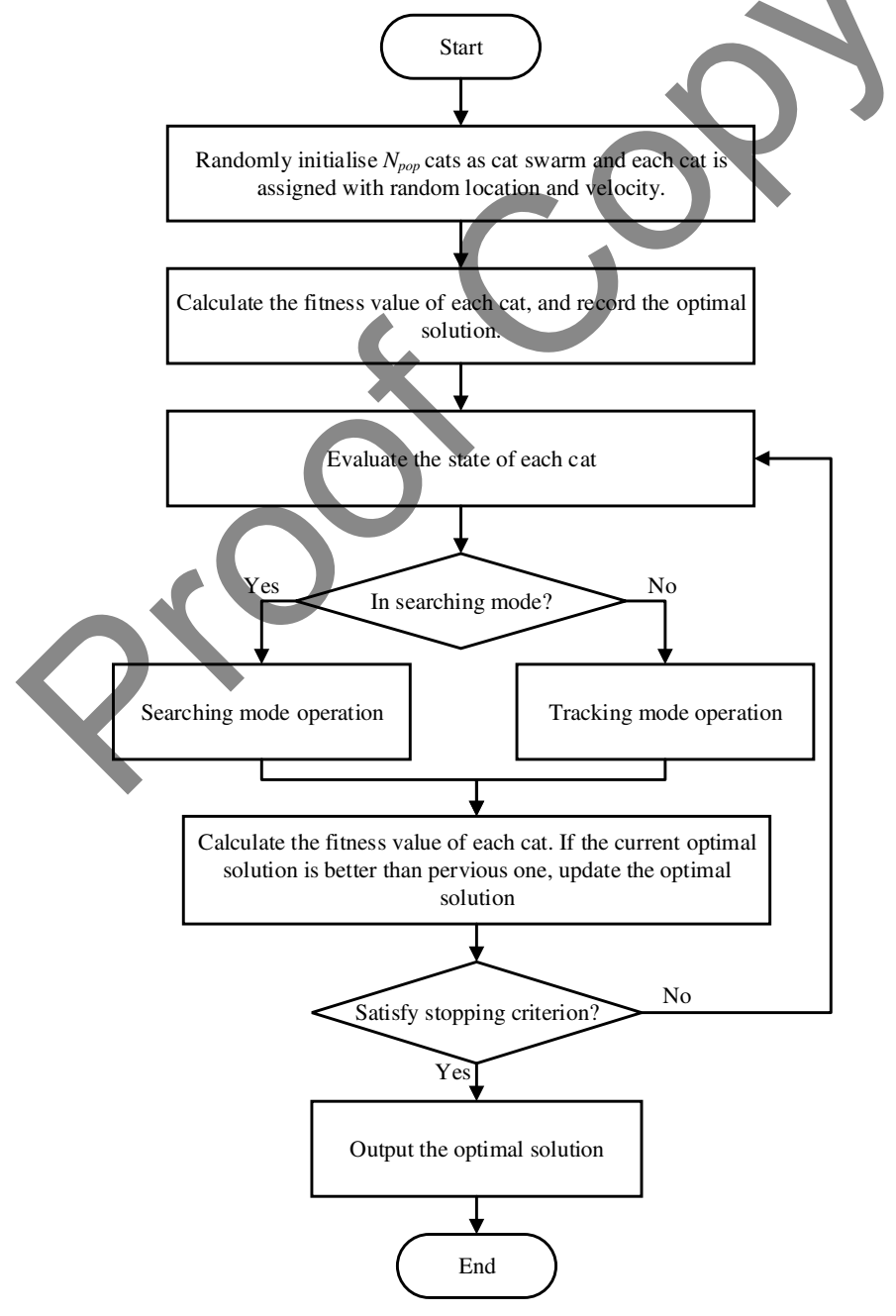

Fig. 3. Flowchart of CSO algorithm. 
the MP, part of cats will turn into the tracking model and the rest will be in the searching mode.

Step 3. Calculate the fitness value of each cat and find out the optimal one. If the current optimal location is better than previous ones, record it into the memory. Otherwise, keep the previous optimal value.

Step 4. The cats move based on the value of their flags. If the cat is in the searching mode, it will be applied to the searching mode procedure; if the cat is in the tracking mode, it will be applied to the tracking mode procedure.

Step 5. Re-assign the cats into the tracking mode or searching mode.

Step 6. Evaluate the stopping criterion. If the stopping criterion is met, the algorithm will be terminated. Or else, go to Step 3 and repeat Steps 3-5 until the criterion is met.

\section{Proposed Method for Modeling MRE Isolator}

In this paper, the ELM and the CSO are combined to contribute to a hybrid model to characterize the nonlinear dynamics of the MRE isolator. In the proposed model, the available inputs include the captured responses and supplying current of the device. However, to achieve the best performance of the ELM model, the model (network) configuration should be determined during the model training, before it is used to predict the shear force of the MRE isolator in application. Generally, the numbers of inputs and hidden layer neurons are two significant parameters that are directly associated with the generalization ability of the ELM. If the numbers of network inputs and hidden layer neurons are too small, the training time is relatively short but the trained network is difficult to capture the effective information from the samples, which means that the network could not summarize the feature patterns of the samples. Inyersely, if the network input and hidden layer neuron are set to the larger values, apart from the long training time, the network may be heavily dependent on some inputs or hidden neurons, which will lead to the problem of overfitting and low generalization capacity. Accordingly, these parameters of the ELM model should be optimized to realise the optimal performance. In this work, optimizing the inputs and the neuron number in the hidden layer can be regarded as solving a discrete optimization problem. The network input selection can be expressed as the assignment of a binary string with the bit values of 0 and 1 , and the length of the string is consistent with the total number of the network inputs. If $k$ th ( $\left.k=1,2, \ldots, N_{\text {input }}\right)$ input is selected by the optimization algorithm, the value of corresponding bit will be set as 1 . Or else, it will be set as 0 . Apart from the network inputs, the neuron number in the hidden layer is another important parameter that should also be considered to be encoded in the string. As a result, in this work, the location of the cat in CSO is defined as a binary string, shown in Fig. 4, in which $L_{\text {input }}$ is the bit length that is employed to encode all the possible inputs and $L_{\text {hidden }}$ 


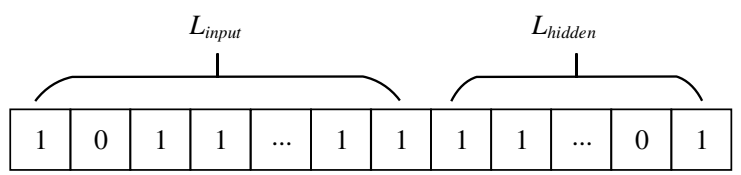

Fig. 4. Binary encoding scheme for the input and hidden layer neuron number of ELM.

denotes the bit length that is employed to encode the neuron number in the hidden layer between minimal and maximal values, with the following relationship.

$$
L_{\text {hidden }}=\left\lceil\log _{2}\left(N_{\text {hidden }}^{\max }-N_{\text {hidden }}^{\min }\right)\right\rceil,
$$

where $N_{\text {hidden }}^{\max }$ and $N_{\text {hidden }}^{\min }$ denote the maximal and minimal values of neuron number in the hidden layer, respectively; the symbol $\lceil\cdot\rceil$ denote the ceil function that returns the number value rounded to the smallest integer.

Furthermore, to solve this discrete optimization problem in this work, the standard CSO should be converted to the binary-coded discrete CSO (BCDCSO). Compared with standard CSO, the BCDCSO slightly changes in the searching mode. Because the values of cat locations in BCDCSO are 1 or 0 , a binary mutation is defined to show the variation of the present location of the cat. Consequently, the mutation operation probability (MOP) is used to substitute for the CRD in standard CSO. In the searching mode, for each copy of SMP, choose CND dimensions and mutate them randomly in accordance with MOP and substitute for the previous ones. In the tracking mode of BCDCSQ, the velocity $\bar{v}_{k, d}$, different from that in the standard CSO, is defined as the mutation probability of the cat at each dimension. And its mathematical expression is given as follows.

$$
\mathcal{v}_{k, d}=\left\{\begin{array}{ll}
v_{k, d}^{0}, & x_{k, d}=1 \\
v_{k, d}^{1}, & x_{k, d}=0
\end{array},\right.
$$

where the vector $v_{k d}^{0}$ denotes the probabilities of the bits that change from 1 to 0 and the vector $v_{k d}^{1}$ denotes the probabilities of the bits that change from 0 to 1 . The location of $k$ th cat at $d$ th dimension is updated as:

$$
\begin{gathered}
x_{k, d}=\left\{\begin{array}{ll}
0, & r_{2} \geq \operatorname{sigm}\left(\bar{v}_{k, d}\right) \\
1, & r_{2}<\operatorname{sigm}\left(\bar{v}_{k, d}\right)
\end{array},\right. \\
\operatorname{sigm}\left(\bar{v}_{k, d}\right)=\frac{1}{1+e^{-\bar{v}_{k, d}}},
\end{gathered}
$$

where $r_{2}$ denotes the random numbers between 0 and 1 ; sigm denotes the sigmoid function, the curve of which is shown in Fig. 5. It is shown that the value of the function approximates to 1 with the higher velocity, and the function value is close to 0 when the velocity is assigned with a lower value.

Then, based on the BCDCSO and ELM, the proposed hybrid learning approach is employed for the nonlinear characterization of the MRE isolator. Here, the Bayesian Information Criterion (BIC) is adopted as the fitness function for the Pareto-based 
Y. Yu et al.

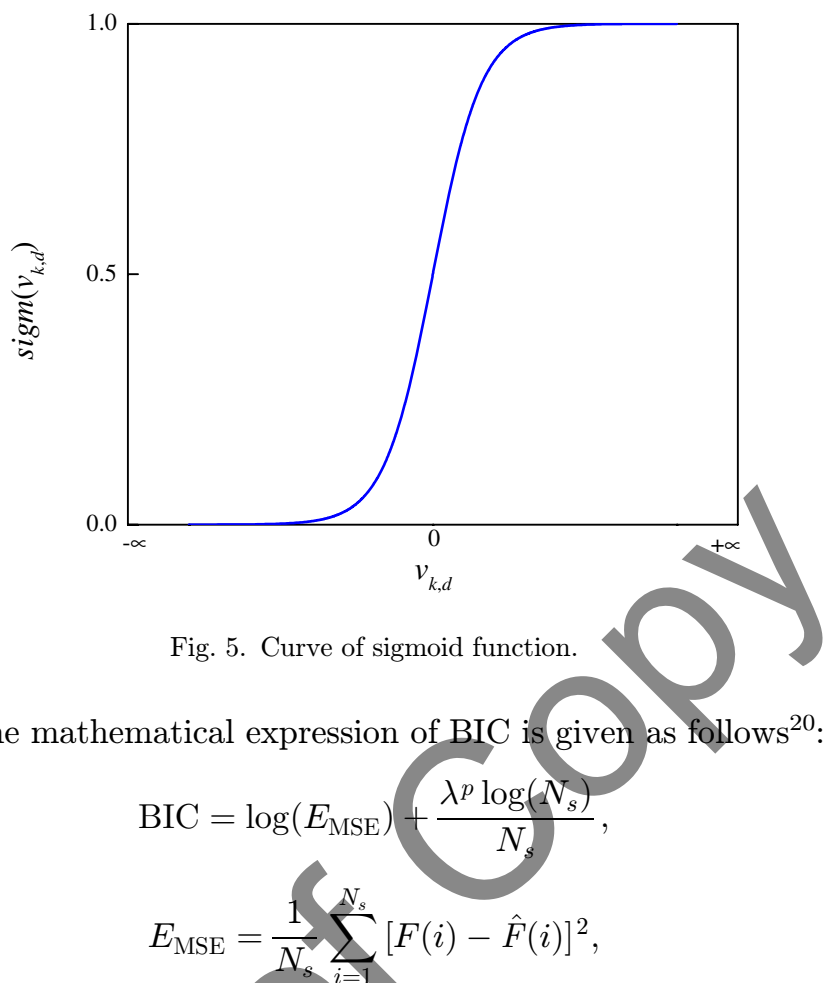

where $E_{\mathrm{MSE}}$ denotes the mean squares errors (MSE) between the experimental results and network outputs: $N_{S}$ denotes the total number of data for model training; $F$ and $\hat{F}$ denote the experimental result and model prediction, respectively; $p$ is an exponent parameter that is used to provide different penalties to the model complexity, and its value is more than 1 for the nonlinear regression; $\lambda$ represents the complexity of the model structure, which is defined as the total parameter number of input and hidden layers. In this study, the designed ELM just has one output neuron, so the value of $\lambda$ can be calculated as:

$$
\lambda=\left(1+N_{\text {input }}\right) \cdot N_{\text {input }}+N_{\text {hidden }},
$$

where $N_{\text {input }}$ and $N_{\text {hidden }}$ denote the neuron numbers in the input and hidden layers, respectively. The function of BIC is to add the penalty to the model complexity so as to get the parsimonious model. Since the fitness value is decreased during the algorithm iteration, the optimal ELM model is selected corresponding to the cat with lowest fitness value.

\section{Dynamical Performance and Network Model Identification of MRE Base Isolator}

To characterize the dynamic performance of the MRE isolator, several groups of experimental testing are conducted in the laboratory under various loading 


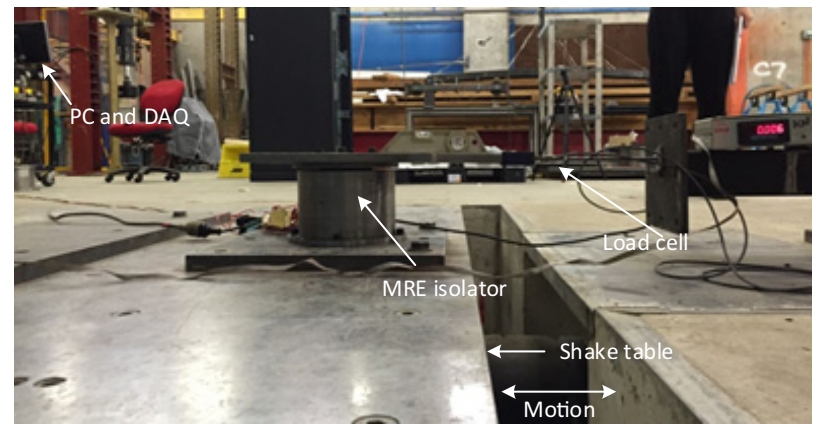

Fig. 6. Dynamical testing setup of the MRE isolator.

conditions. In this study, only horizontal loading is considered for experimental tests, because the device is used to change the lateral stiffness of the isolated structure and related studies show that the vertical loading has little impact on the dynamic performance of the isolator. Figure 6 gives the equipment used for the testing of the MRE isolator. A hydraulic vibration table is used to provide the horizontal loading signals for the device, which is fixed on the table and moves with the movement of the vibration table. The displacement sensor, internally connected with the platform of the vibration system, is used to obtain the displacement responses of the device. A force sensor, connected with the top of the isolator, is used to measure the force responses generated. The DC power is employed to energize the magnetic coils of the device, which is able to offer the electricity currents from $0 \mathrm{~A}$ to $3 \mathrm{~A}$ with the increment of $1 \mathrm{~A}$. A desktop with aDAQ system is used to record both loading and response signals.

To fully portray the nonlinear dynamics of the MRE isolator, three types of loading conditions are considered in this study: sinusoid loading, random loading, and seismic loading. For the sinusoid loading, the loading amplitude ranges from $2 \mathrm{~mm}$ to $8 \mathrm{~mm}$ with the frequency of $[1,4] \mathrm{Hz}$. For the random loading, the maximal amplitude is set as $5 \mathrm{~mm}$ and the varied loading frequency is in the range of $[1,20] \mathrm{Hz}$. For the seismic loading, El-Centro earthquake records are used as excitation inputs to drive the device. In this earthquake, the N-S component recorded at the Imperial Valley Irrigation District substation in El-Centro, California, during the earthquake on May 19, 1940. To avoid the deformation of the isolator under critical level, the seismic inputs are scaled with the maximal amplitude of $4 \mathrm{~mm}$. The sampling rate is set as $256 \mathrm{~Hz}$. To guarantee the stable performance of the device, more than two cycles of responses will be captured for each loading condition. Table 1 summarizes the testing conditions for the MRE isolator.

The overall structure for model identification of the MRE isolator is shown in Fig. 7. As mentioned in the previous section, the current and previous displacement and velocity responses, present current level and previous force responses are suggested as the network inputs while the generated shear force is selected as the 
Table 1. Summary of testing conditions (C: Case).

\begin{tabular}{|c|c|c|c|c|c|c|c|c|c|c|c|}
\hline \multirow[b]{4}{*}{ Cur } & \multicolumn{11}{|c|}{ Loading condition } \\
\hline & \multicolumn{9}{|c|}{$\operatorname{Sin}$} & \multirow[b]{3}{*}{ Ran } & \multirow[b]{3}{*}{ Sei } \\
\hline & \multicolumn{3}{|c|}{$1 \mathrm{~Hz}$} & \multicolumn{3}{|c|}{$2 \mathrm{~Hz}$} & \multicolumn{3}{|c|}{$4 \mathrm{~Hz}$} & & \\
\hline & $2 \mathrm{~mm}$ & $4 \mathrm{~mm}$ & $8 \mathrm{~mm}$ & $2 \mathrm{~mm}$ & $4 \mathrm{~mm}$ & $8 \mathrm{~mm}$ & $2 \mathrm{~mm}$ & $4 \mathrm{~mm}$ & $8 \mathrm{~mm}$ & & \\
\hline $0 \mathrm{~A}$ & $\mathrm{C} 1$ & C 5 & C 9 & C 13 & C 17 & C 21 & C 25 & C 29 & C 33 & C 37 & C 41 \\
\hline $1 \mathrm{~A}$ & $\mathrm{C} 2$ & C 6 & C 10 & C 14 & C 18 & C 22 & C 26 & C 30 & C 34 & C 38 & C 42 \\
\hline $2 \mathrm{~A}$ & C 3 & C 7 & C 11 & C 15 & C 19 & C 23 & C 27 & C 31 & C 35 & C 39 & C 43 \\
\hline $3 \mathrm{~A}$ & C 4 & C 8 & C 12 & C 16 & C 20 & C 24 & C 28 & C 32 & C 36 & C 40 & C 44 \\
\hline
\end{tabular}

Note: Cur (Current), Sin (Sinusoid), Ran (Random), Sei (Seismic).

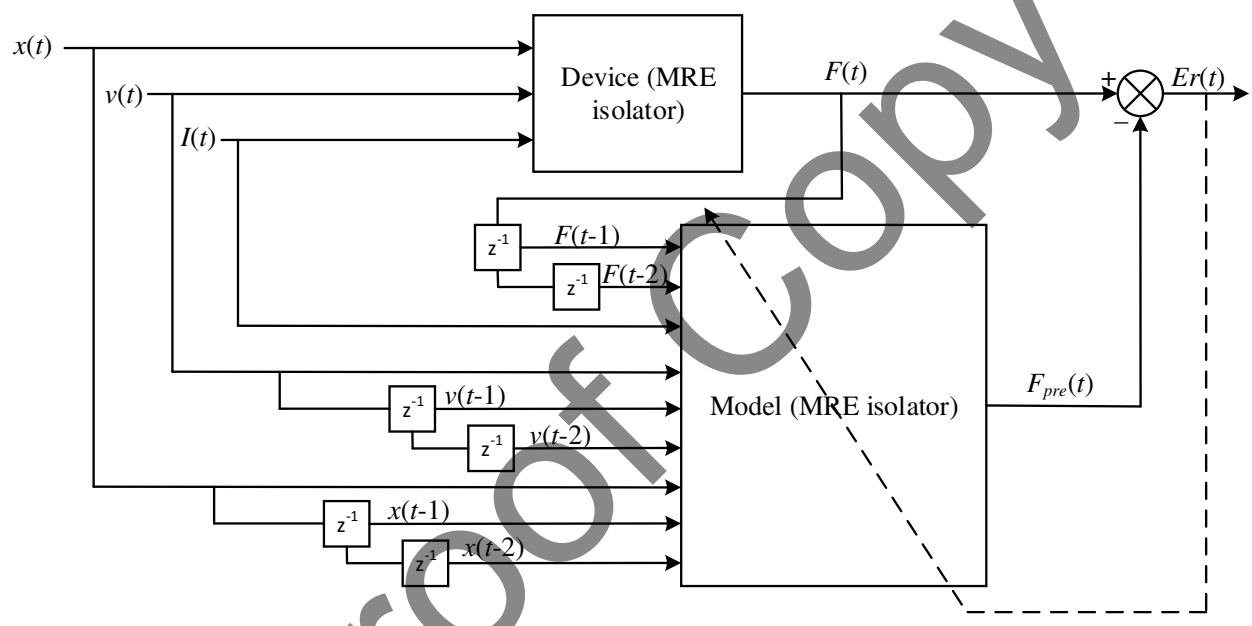

Fig. 7. Identification scheme of the BCDCSO-ELM model for the MRE isolator.

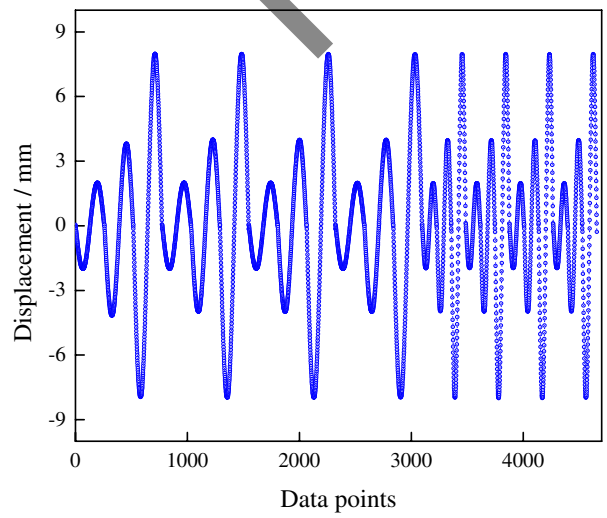

(a)

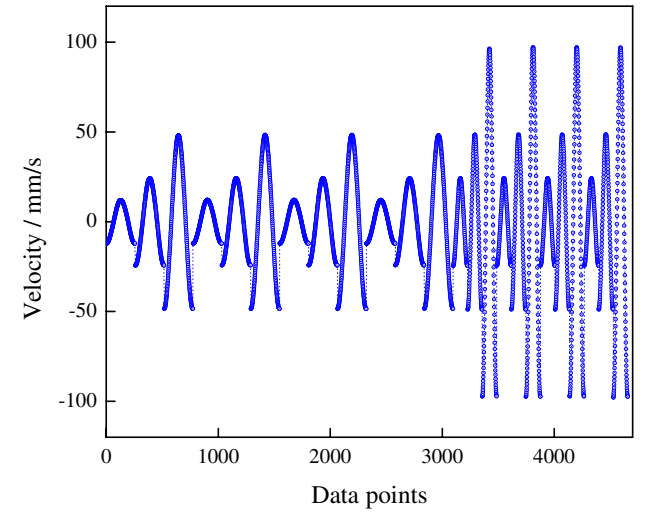

(b)

Fig. 8. Example of training data: (a) displacement, (b) velocity, (c) current, and (d) shear force. 


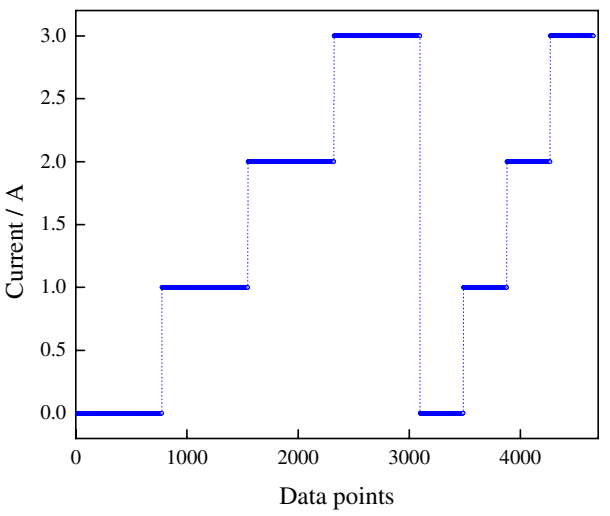

(c)

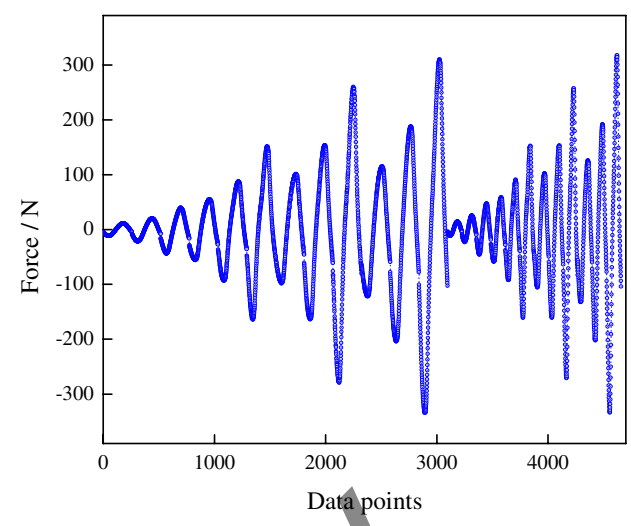

Fig. 8. (Continued)

network output. All the measurement data are divided into two groups: one group data from Cases 1 to 24 are used as training samples to set up the MRE isolator model; the other group of data from Cases 25 to 44 are used as the validation samples to test the performance of the trained model. Figure 8 gives the example of training data of displacement, velocity, current and shear force of the MRE isolator.

\section{Results and Discussions}

In this work, the BCDCSO and the ELM algorithms are implemented based on Matlab v.2015a running on a laptop with Intel Core i7 processor and 8 GB memory. The parameter setting of BCDCSO is given as: swam size (cat population) $N_{\text {pop }}=50$, maximum iteration number $N_{\text {it }}=400$, searching memory pool $\mathrm{smp}=5$, mixture probability $\mathrm{mp}=20 \%$, mutation operation probability mop $=40 \%$, change number of dimensions cnd $=80 \%$, and $c_{1}=2$. For the fitness function, the model complexity parameter $p=2$, as suggested in 20 . To effectively decrease the influence of multi-dimensional network input on model accuracy, a normalization operation is conducted on all the experimental data. The equation for data normalization is given as:

$$
\tilde{y}(i)=\left[y(i)-y_{\min }\right] /\left[y_{\max }-y_{\min }\right]
$$

where $y_{\max }$ and $y_{\min }$ denote the maximal and minimal values in the experimental data, and $\tilde{y}$ denotes the normalized result. After this operation, all the elements in the input vector will be scaled in the range of $[0,1]$. Next, the training samples (Cases 1-24) are inputted into the ELM for optimizing the network parameters via the BCDCSO algorithm. In addition to the network inputs illustrated in the Sec. 3, the scope of the neuron number in the hidden layer should be determined before the 
network optimization. According to Ref. 21, the scale of optimal hidden layer neuron number can be calculated as follows:

$$
N_{\text {hidden }}=\sqrt{N_{\text {input }}+N_{\text {output }}}+\zeta
$$

where $N_{\text {input }}$ and $N_{\text {output }}$ denote network input and output numbers, respectively. In this study, the value of $N_{\text {input }}$ is its maximal possible number, i.e. $N_{\text {input }}=9 . \zeta$ is a constant in the range of $[0,9]$. Hence, the scale for optimal hidden layer neuron number is $[4,12]$.

The optimal network parameters are gained through the minimization of the fitness function. The corresponding results are shown in Fig. 9. It is noticeable that when the input number is small, the BIC error is around 9. Then, the BIC has an obvious decrease with the adding input number. When the input number exceeds a specific value (6), the value of BIC tends to be stable and the change is not apparent. Similarly, after the neuron number in the hidden layer arrives at 10, the network has the optimal BIC. This optimal design contributes the ELM-based model with six inputs and 10 hidden neurons with the BIC of 4.7042. The six inputs are current and previous displacement responses $x(t)$ and $x(t-2)$, previous velocity responses $v(t-1)$ and $v(t-2)$, current electricity current level $I(t)$ and previous force response $F(t-1)$, respectively.

Then, based on the optimal model configuration, the samples from Cases 25 to 44 are employed to make up the validation set to evaluate the performance of the trained model. Figures 10 and 11 exhibit the capacity of the proposed model to predict the hysteresis characteristics of the device, in which Fig. 10 shows the model performance to portray the current-dependent property of the device and Fig. 11 demonstrates the model performance to forecast the excitation-amplitude property of the device. It can be observed from Fig. 10 that with increase of the applied

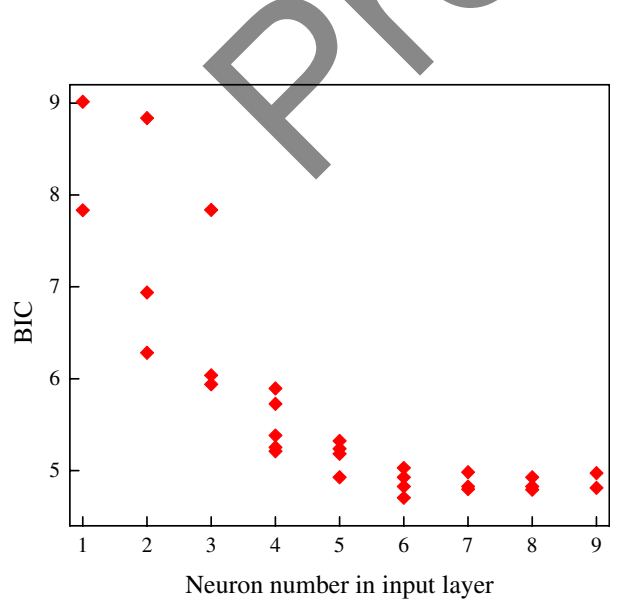

(a)

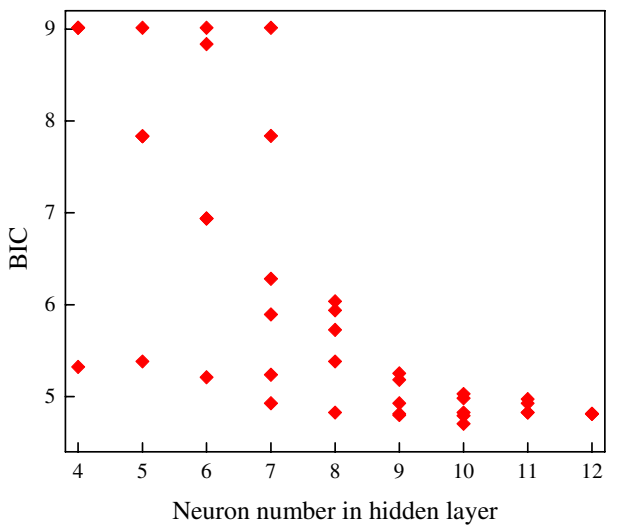

(b)

Fig. 9. BIC values of trained network with different parameters: (a) input number and (b) hidden layer neuron number. 


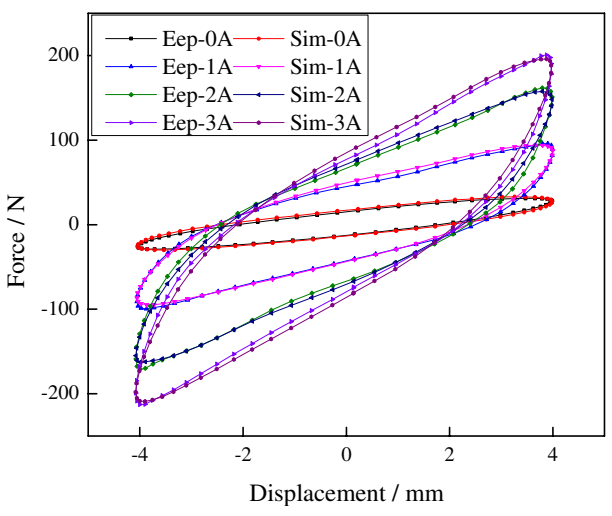

(a)

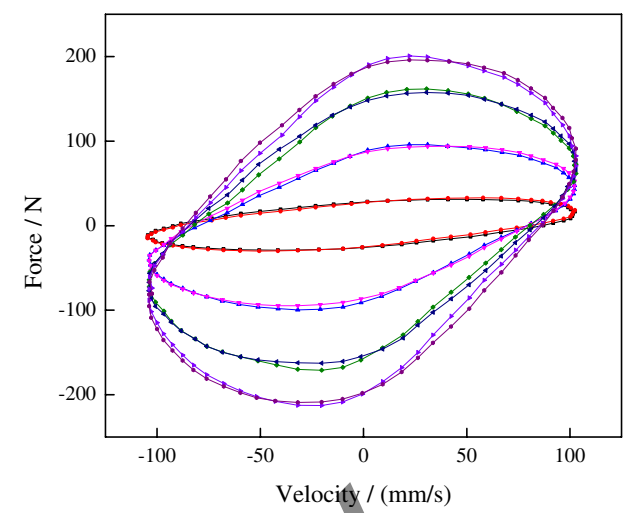

(b)

Fig. 10. Response comparison with different applied current levels: (a) force-displacement response and (b) force-velocity response (Exp: experimental; Sim: simulated).

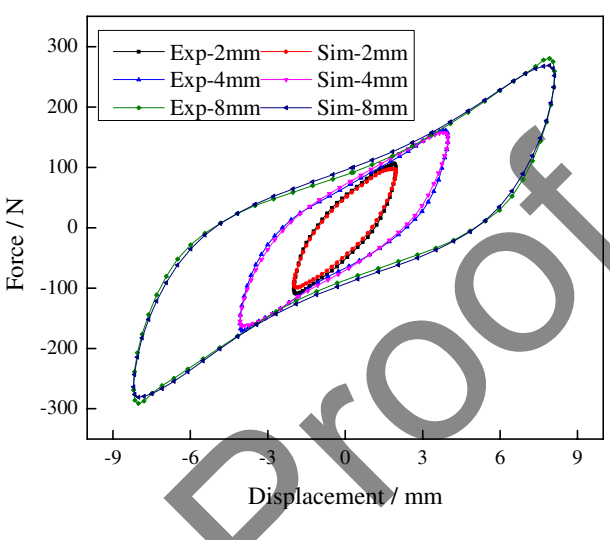

(a)

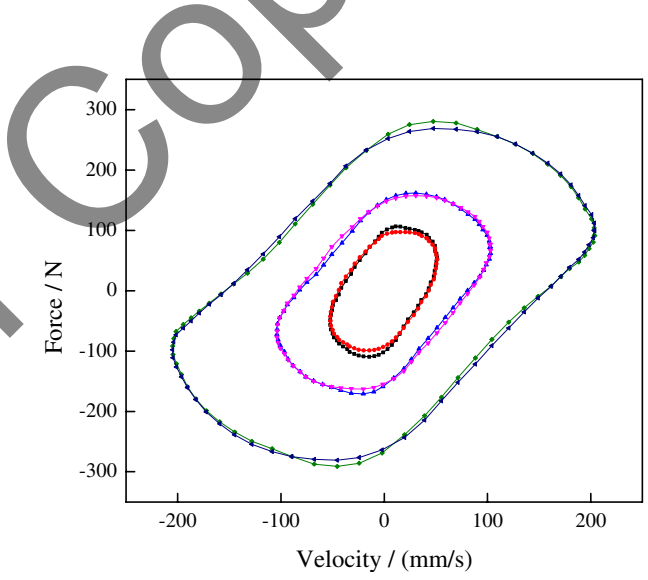

(b)

Fig. 11. Response comparison with different excitation amplitudes: (a) force-displacement response and (b) force-velocity response (Exp: experimental; Sim: simulated).

current, the effective stiffness of the isolator will be increased simultaneously, which is represented by the slope of the force-displacement loop. This phenomenon makes the MRE isolator more adaptive to be applied in the base isolation system via the change of the stiffness property. The good match between experimental results and model predictions validates the effectiveness of the proposed model to characterize this feature. On the other hand, different from current-dependent property in Figs. 10 and 11 shows that the stiffness of the device has a slight deterioration with the increase of the loading amplitude from $2 \mathrm{~mm}$ to $8 \mathrm{~mm}$. This phenomenon is also called Murphy's effect. The comparison results in Fig. 11 effectively demonstrate the capacity of the proposed model to predict this characteristic. 


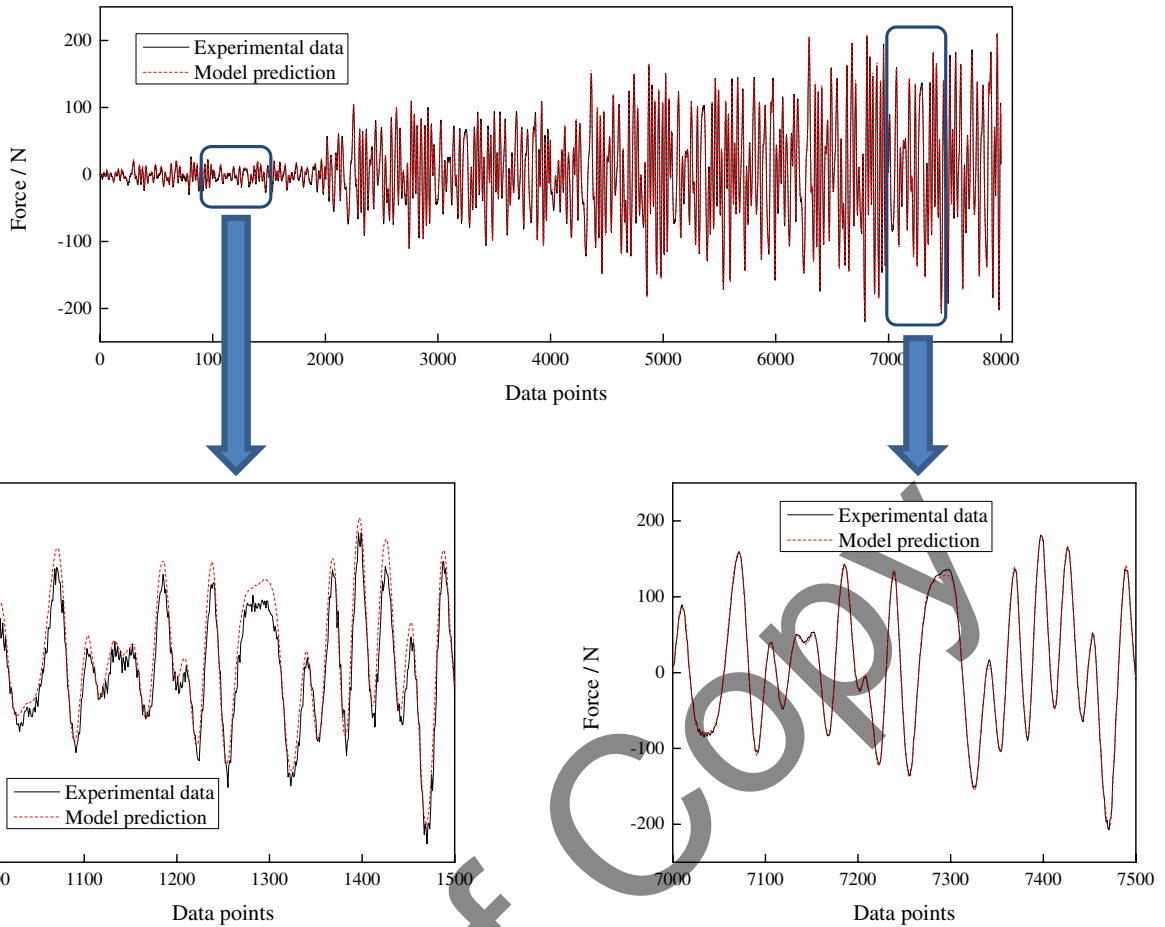

Fig. 12. Response comparison with random excitation and different applied currents.

Figures 12 and 13 display the comparison results between experimental data and model outputs when the MRE isolator is driven by random and seismic excitations and supplied with four levels of electricity currents. The results in both figures show that the proposed model is capable of providing good performance to adapt the changeable loading frequency. Even though some obvious deviations (circled in the figure) appear at the peak areas, the model well forecasts the changeable tendency of the shear force, which indicates the promising application of the BCDCSO-ELM model in the development of the semi-active controller of the device.

To further elaborate the effectiveness of BCDCSO to improve the performance of the ELM model, a comparative study is conducted based on same training and validation samples. The correlation coefficient $C$ between the experimental results and network outputs is employed as the evaluation index. The mathematical expression of the correlation coefficient is shown as follows ${ }^{22}$ :

$$
C=\frac{\sum_{k=1}^{N_{t}}\left[F(i)-F_{m}(i)\right] \cdot\left[\hat{F}(i)-\hat{F}_{m}(i)\right]}{\sqrt{\sum_{k=1}^{N_{t}}\left[F(i)-F_{m}(i)\right]^{2}} \cdot \sqrt{\sum_{k=1}^{N_{t}}\left[\hat{F}(i)-\hat{F}_{m}(i)\right]^{2}}},
$$

where $N_{t}$ denotes the total number of testing samples; $F_{m}$ and $\hat{F}_{m}$ denote the mean values of experimental results and corresponding network outputs, respectively. The 


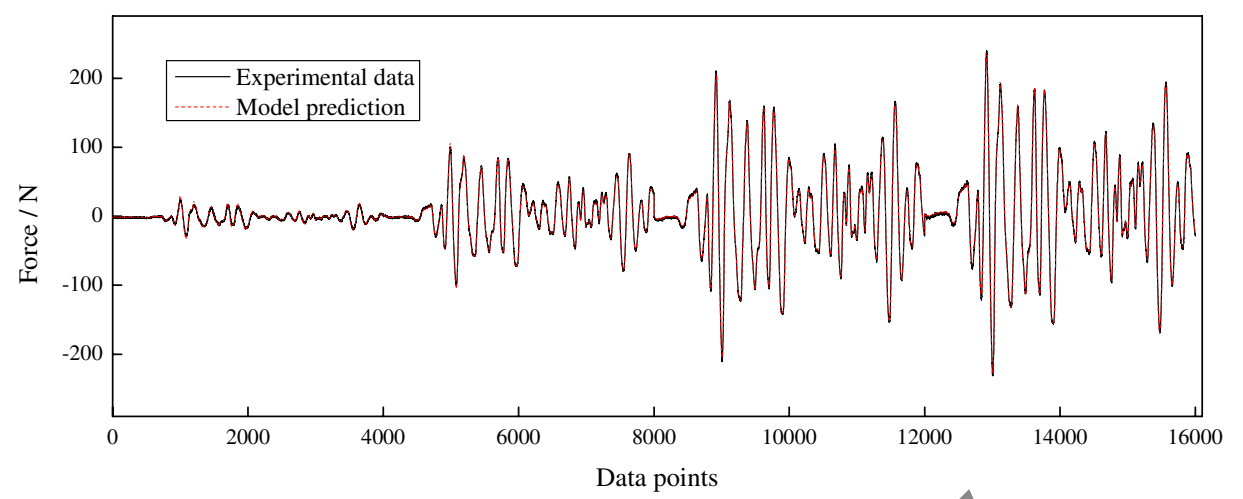

Fig. 13. Response comparison with seismic excitation and different applied currents.

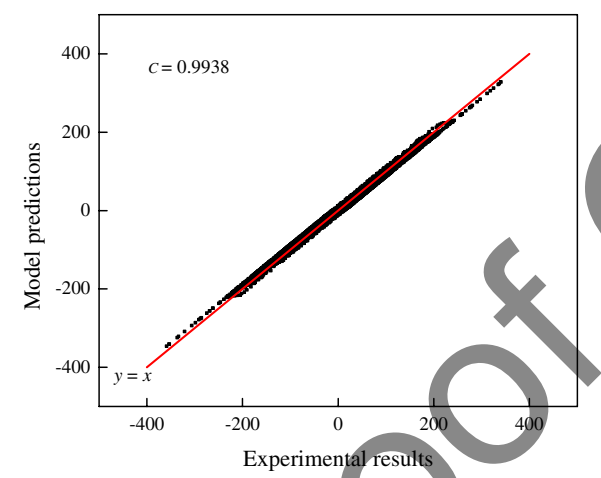

(a)

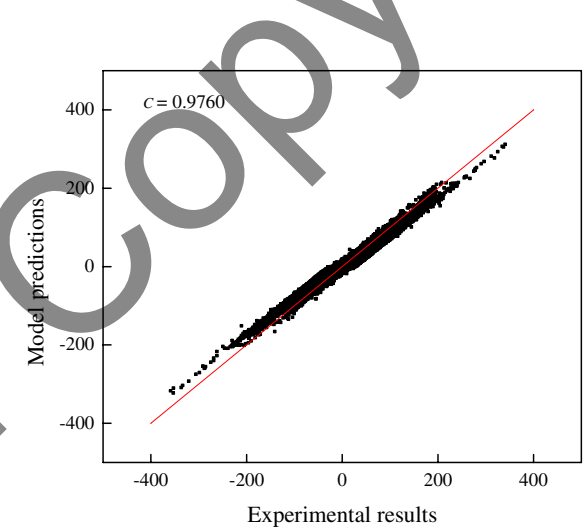

(b)

Fig. 14. Correlation analysis between validation samples and network outputs: (a) BCDCSO-ELM and (b) ELM.

correlation coefficient is a good indicator to represent the fitting degree between two sets of data sequences. Generally, a high correlation coefficient indicates good agreement of two sequences. Figure 14 compares the correlation coefficients between the experimental results and outputs from two models: BCDCSO optimized ELM and standard ELM. It can be concluded from the figure that the values of the correlation coefficients are 0.9938 and 0.9760 for BCDCSO-ELM and ELM, respectively. Thus, the optimized ELM has better generalization ability than the ELM without optimization in terms of nonlinear characterization of the MRE isolator. The superiority of BCDCSO-ELM over ELM is further verified.

As all we known, in practice the measured responses are inevitably contaminated with background noises. On this occasion, it is definitely necessary to assess the robustness of the proposed BCDCSO-ELM model under external noise influence. In this case, the white Gaussian noise is employed to be added into the current signals 
for the simulation of noisy data measurements. In this work, the effect of three different levels of noise on the performance of the trained BCDCSO-ELM model is investigated. In the meantime, a comparative study between the BCDCSO-ELM model and standard ELM model is conducted to illustrate the noise resist characteristics of the proposed model.

Figure 15, respectively, gives the noisy current signals with three types of signal to noise $(\mathrm{S} / \mathrm{N})$ ratios, i.e. 100,50 and $20 \mathrm{~dB}$. The MSEs between noisy signals and the signals without noises are 3.6e-10, 3.5e-5, and 0.0111, respectively. Figures 16-18 provide the correlation results between the BCDCSO-ELM model and standard model, which are used to indicate the effect of measurement noise on the robustness of the model. From the results, it is clearly observed that the noise disturbance has little impact on the generalization ability of the BCDCSO-ELM model, which is able to maintain the high performance $(C=0.9702)$ even if the noise level can arrive at $20 \mathrm{~dB}$ S/N ratio. On the contrary, the standard ELM model is relatively vulnerable to the external noise influence and the values of its correlation results have an obvious decrease with the increase of the $\mathrm{S} / \mathrm{N}$ ratio. As a consequence, it is

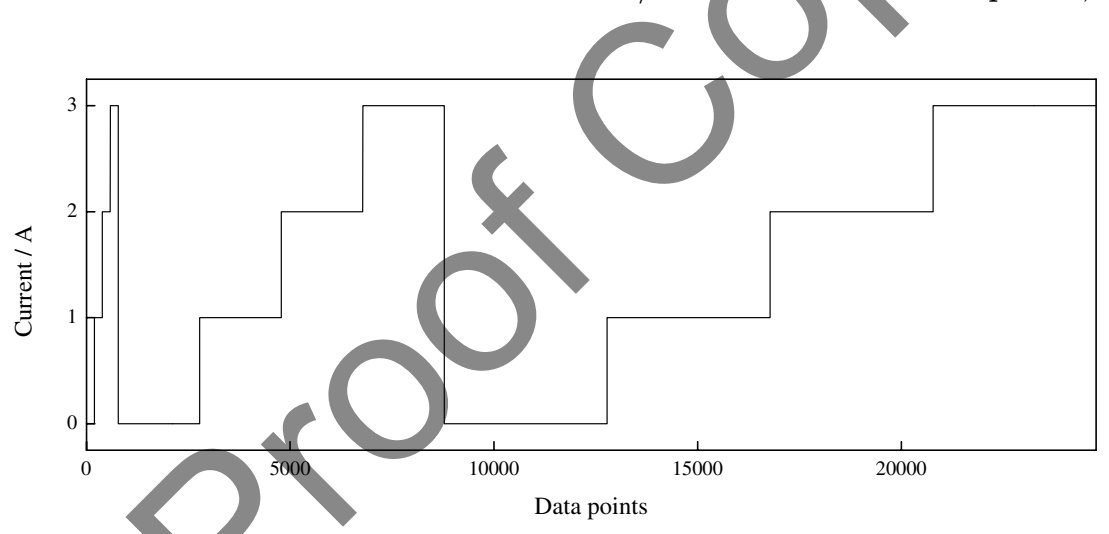

(a)

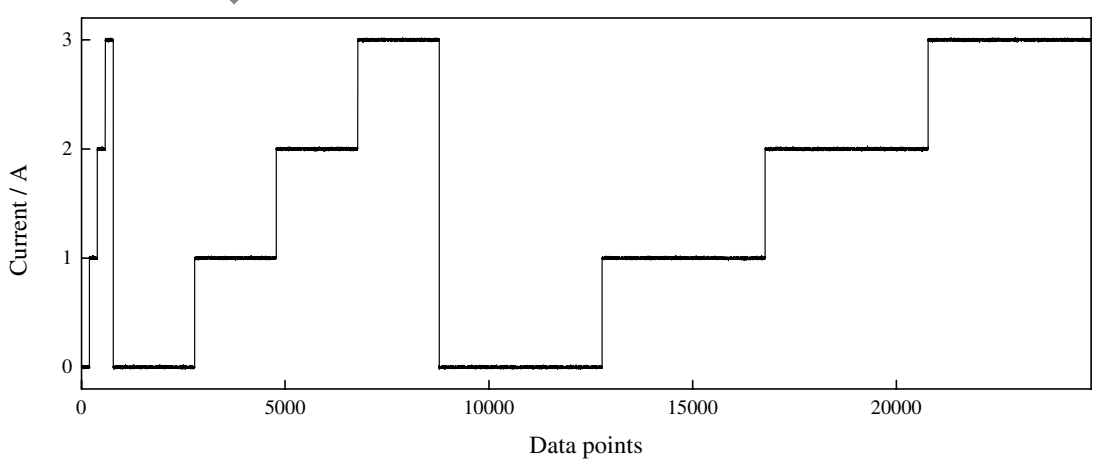

(b)

Fig. 15. Applied currents with different ratios of white Gaussian noise: (a) $100 \mathrm{~dB}$, (b) $50 \mathrm{~dB}$ and (c) $20 \mathrm{~dB}$. 


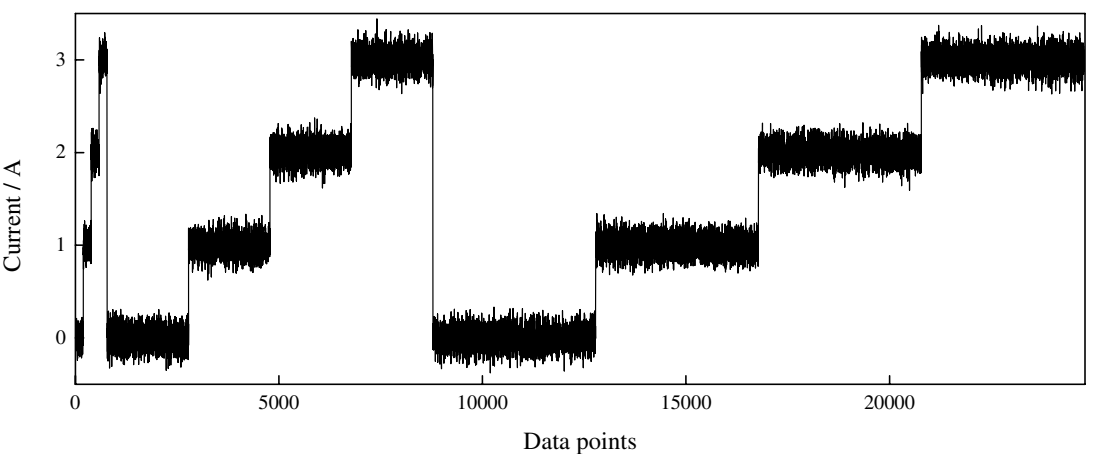

(c)

Fig. 15. (Continued)

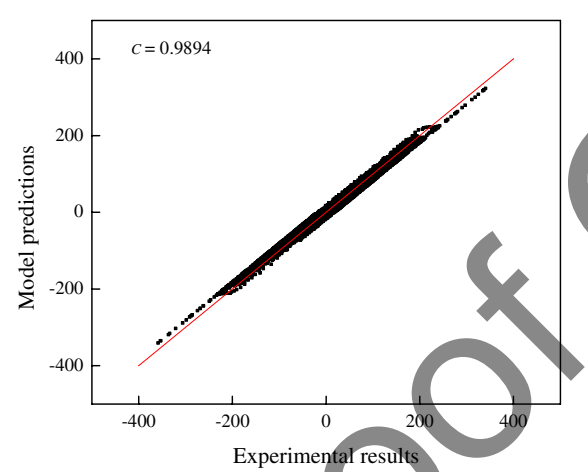

(a)

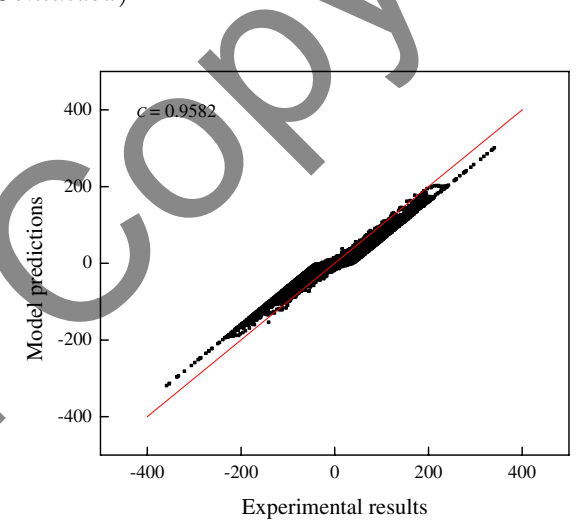

(b)

Fig. 16. Correlation analysis between validation samples and network outputs with $100 \mathrm{~dB} \mathrm{~S} / \mathrm{N}$ ratio white Gaussian noises: (a) BCDCSO-ELM and (b) ELM.

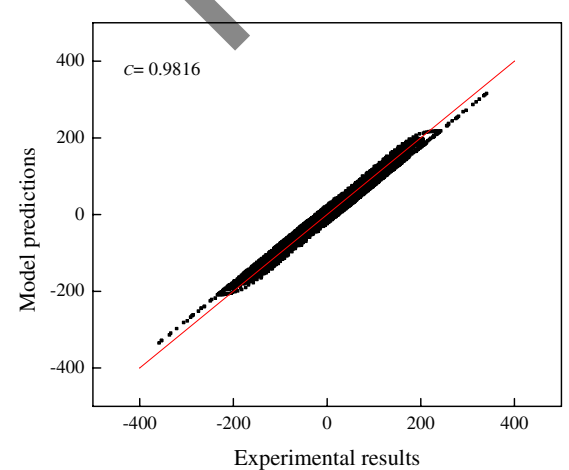

(a)

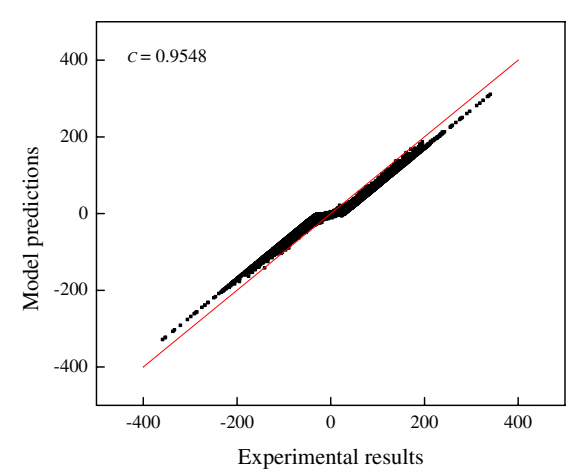

(b)

Fig. 17. Correlation analysis between validation samples and network outputs with $50 \mathrm{~dB} \mathrm{~S} / \mathrm{N}$ ratio white Gaussian noises: (a) BCDCSO-ELM and (b) ELM. 


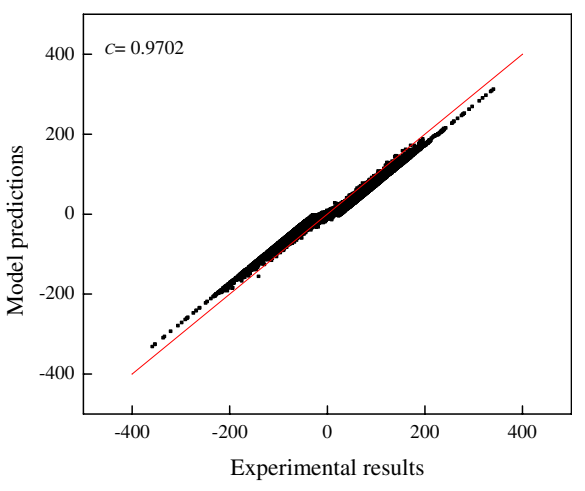

(a)

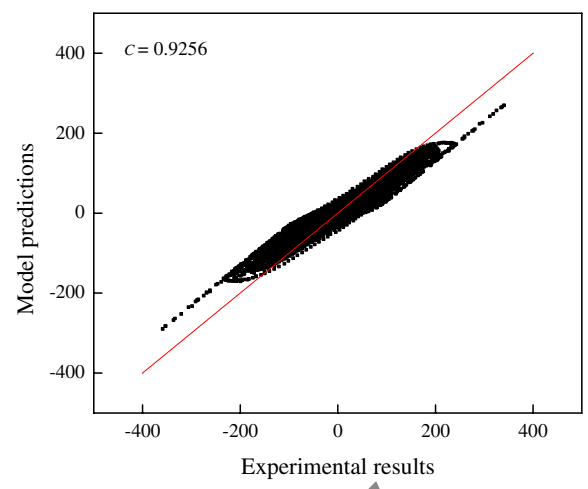

(b)

Fig. 18. Correlation analysis between validation samples and network outputs with $20 \mathrm{~dB} \mathrm{~S} / \mathrm{N}$ white Gaussian noises: (a) BCDCSO-ELM and (b) ELM.

summarized that the BCDCSO-ELM model is capable of providing not only good generalization performance but also outstanding robustness, which well satisfies the requirements in the practical applications.

\section{Conclusions}

This paper presents a novel ELM-based model to characterize the nonlinear dynamics of the MRE isolator, an adaptive smart device used in the civil structures for the mitigation of hazard vibration responses. To achieve the optimal generalization capacity of the proposed model, an improved CSO algorithm is employed to select the best network inputs and neuron number in the hidden layer. The performance of the proposed method is evaluated based on the displacement, velocity, shear force, and applied current responses captured from a prototype of the MRE isolator. The results show that the ELM with six input neurons and 10 hidden neurons has the best fitness values (BIC) for modeling the MRE isolator, which is superior to the standard ELM without optimal parameters in terms of the correlation coefficients between experimental results and model predictions. Finally, the robustness of the proposed model is also investigated via evaluating the effect of noisy current signals on the model accuracy. The analysis results show that the proposed model has strong noise-abatement property, which is able to improve the control effects of existing MRE-based isolation systems with noise disturbance.

\section{Acknowledgments}

This work was supported by Australian Research Council via the Discovery Project (No. DP150102636). The authors would like to thank the financial support from the funding body. 


\section{References}

1. Fuchs, J. Sutrisno, F. Gordaninejad, M. B. Caglar and Y. M. Liu, Surface polymerization of iron particles for magnetorheological elastomer, J. Appl. Polym. Sci. 117(2) (2010) $934-942$.

2. M. Farshad and A. Benine, Magnetoactive elastomer composites, Polym. Test. 23(3) (2004) 247-253.

3. Y. Fan, X. Gong, S. Xuan, L. Qin and X. Li, Effect of cross-link density of the matrix on the damping properties of magnetorheological elastomers, Indust. Eng. Chem. Res. 52(2) (2013) 771-778.

4. Q. Ha, S. Royel, J. Li and Y. Li, Hysteresis modeling of smart structure MR devices using describing functions, IEEE-ASME T Mech. 21(1) (2016) 44-50.

5. Y. Yu, S. Royel, J. Li, Y. Li and Q. Ha, Magnetorheological elastomer base isolator for earthquake response mitigation on building structures: Modeling and second-order sliding mode control, Earthq. Struct. 11(6) (2016) 943-966.

6. N. Hoang, N. Zhang and H. Du, A dynamic absorber with a soft magnetorheological elastomer for powertrain vibration suppression, Smart Mater. Struct. 18(7) (2009) 074009.

7. N. Hoang, N. Zhang, W. H. Li and H. Du, Development of a torsional dynamic absorber using a magnetorheological elastomer for vibration reduction of a powertrain test rig, $J$. Intell. Mater. Syst. Struct. 24(16) (2013) 2036-2044.

8. J. M. Ginder, M. E. Nochols, L. D. Elie and S. M. Clark, Controllable-stiffness components based on magnetorheological elastomers, smart structures and material 2000: Smart structures and integrated systems, Proc. SPIE 3985, (2000) 418-425.

9. H. Du, W. Li and N. Zhang, Semi-active variable stiffness vibration control of vehicle seat suspension using an MR elastomer isolator, Smart Mater. Struct. 20(10) (2011) 105003.

10. S. S. Sun, J. Yang, H. X. Deng, H. Du, W. H. Li, G. Alici and M. Nakano, Horizontal vibration reduction of a seat suspension using negative changing stiffness magnetorheological elastomer isolators, Int. J. Vehicle Design 68(1/2/3) (2015) 104-118.

11. Y. Li, J. Li, W. Li and B. Samali, Development and characterization of a magnetorheological elastomer based adaptive seismic isolator, Smart. Mater. Struct. 22(3) (2013) 035005.

12. Y. Li and J. Li, A highly adjustable base isolator utilizing magnetorheological elastomer: Experimental testing and modeling, J. Vib. Acoust. 137(1) (2015) 011009.

13. C. Y. Yang, J. Fu, M. Yu, X. Zheng and B. X. Ju, A new magnetorheological elastomer isolator in shear-compression mixed mode, J. Intel. Mat. Syst. Str. 26(10) (2015) 12901300 .

14. J. Yang, H. Du, W. Li, Y. Li, J. Li, S. Sun and H. X. Deng, Experimental study and modeling of a novel magnetorheological elastomer isolator, Smart Mater. Struct. 22(11) (2013) 117001.

15. M. Behrooz, X. Wang and F. Gordaninejad, Modeling of a new semi-active/passive magnetorheological elastomer isolator, Smart Mater. Struct. 23(4) (2014) 045013.

16. J. Fu, G. Liao, M. Yu, P. Li and J. Lai, NARX neural network modeling and robustness analysis of magnetorheological elastomer isolator, Smart Mater. Struct. 25(12) (2016) 125019.

17. G. B. Huang, Q. Y. Zhu and C. K. Siew, Extreme learning machine: Theory and applications, Neurocomputing 70(1-3) (2006) 489-501.

18. S.S. Kourehli, Application of extreme learning machine to damage detection of plate-like structures, Int. J. Struct. Stab. Dy. (2016) $1750068_{\text {, }}$

19. S. C. Chu and P. W. Tsai, Computational intelligence based on the behaviour of cats, Int. J. Innov. Comput. I. 3 (2007) 163-173.

\begin{tabular}{l} 
AQ: Please \\
provide \\
complete \\
details. \\
\hline
\end{tabular}

details. 
20. M. Qi and G. P. Zhang, An investigation of model selection criteria for neural network time series forecasting, Eur. J. Oper. Res. 132(3) (2001) 666-680.

21. S. S. Kourehli, Damage assessment in structures using incomplete modal data and artificial neural network, Int. J. Struct. Stab. Dyn. 15(6) (2015) 1450087.

22. Y. Yu, Y. Li, J. Li and X. Gu, Self-adaptive step fruit fly algorithm optimized support vector regression model for dynamic response prediction of magnetorheological elastomer base isolator, Neurocomputing 211 (2016) 41-52.

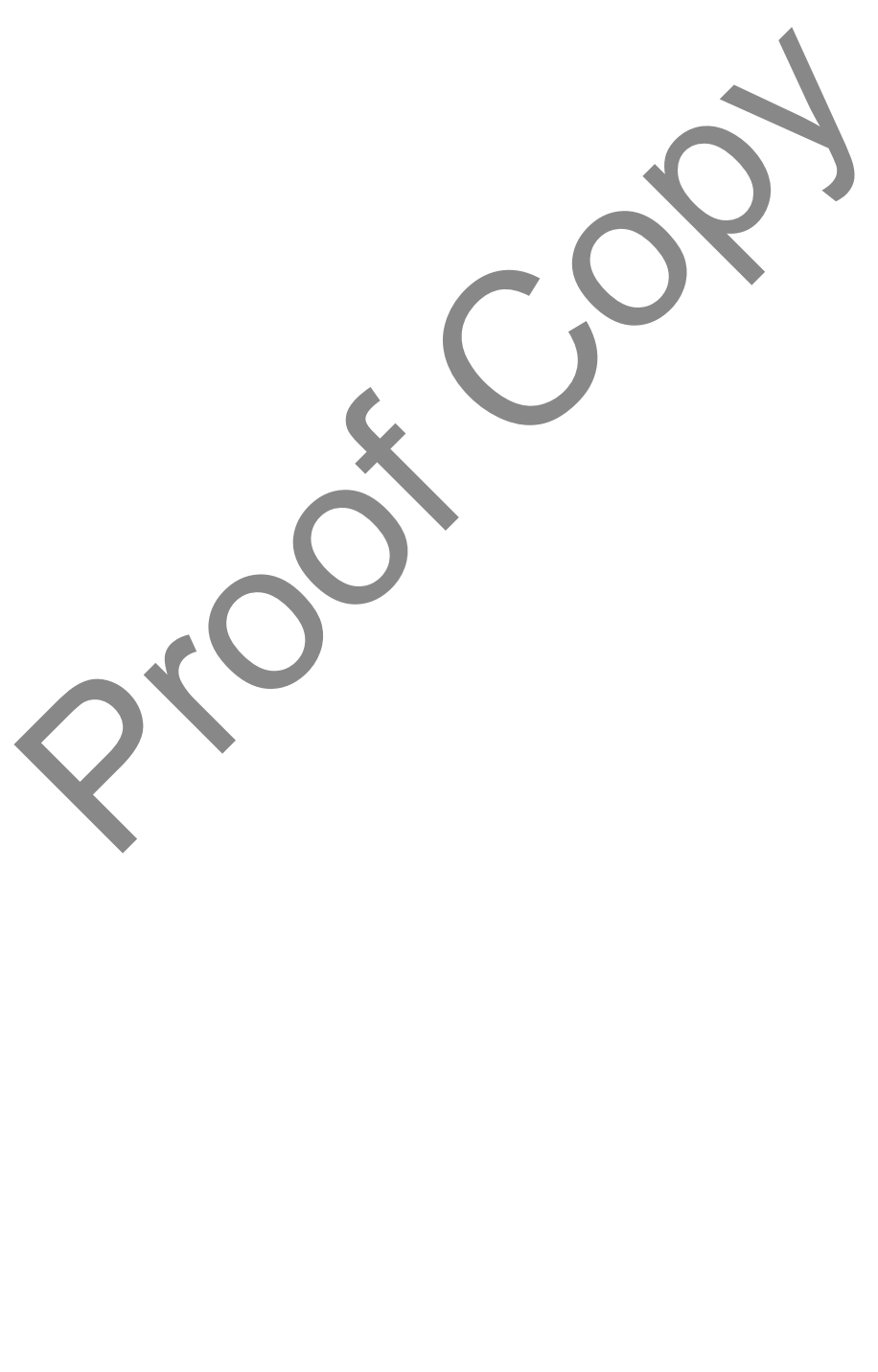

